\title{
Living Donor Liver Transplantation During the COVID-19 Pandemic: an Evolving Challenge
}

\author{
Abu Bakar Hafeez Bhatti ${ }^{1,2}$ (1) Malka Nazish ${ }^{1} \cdot$ Nusrat Yar Khan ${ }^{1} \cdot$ Fazal Manan $^{3} \cdot$ Haseeb Haider Zia ${ }^{1} \cdot$ Abid llyas $^{4}$. \\ Wasib Ishtiaq ${ }^{4}$ Nasir Ayub Khan ${ }^{5}$
}

Received: 6 April 2021 / Accepted: 24 May 2021 / Published online: 15 June 2021

(C) 2021 The Society for Surgery of the Alimentary Tract

\begin{abstract}
Background Maintaining standards of living donor liver transplantation (LDLT) can be a challenge during the corona virus disease 2019 (COVID-19) pandemic. Center-specific protocols have been developed and transplant societies propose limiting elective LDLT. We have looked at outcomes of LDLT during the pandemic in an exclusively LDLT center.

Methods Patients were grouped into pre-COVID (January 2019-February 2020) $(\mathrm{n}=162)$ and COVID (March 2020-January 2021) $(n=53)$ cohorts. We looked at patient characteristics, 30-day morbidity, and mortality. Outcomes were also assessed in donors and recipients who underwent surgery after recovery from COVID-19.

Results The average number of transplants reduced from 11.5/month to 4.8/month. Fewer patients with MELD > 20 underwent LDLT in the COVID cohort (41.3\% versus $24.5 \%, \mathrm{P}=0.03$ ). Out of nine patients with a positive pretransplant COVID-19 PCR, there were $2(22.3 \%)$ deaths on the waiting list. Seven patients underwent LT after recovery from COVID-19 with one 30-day mortality due to biliary sepsis. Three donors with positive COVID-19 PCR underwent uneventful donation after testing negative for COVID-19. No significant difference in 30-day survival was observed in the pre-COVID and COVID cohorts (93.2\% versus 90.6\%) $(\mathrm{P}=0.3)$. Out of two recipients who developed COVID-19 pneumonia within 30 days after LT, there was one mortality. The 1-year survival for the entire cohort with a MELD cutoff of 20 was $90 \%$ and $84 \%(\mathrm{P}=0.2)$.

Conclusion Despite comparable outcomes, fewer sick patients might undergo LDLT during the pandemic. Individuals recovered from COVID-19 might be safely considered for donation or transplantation.
\end{abstract}

Keywords Living donor liver transplantation $\cdot$ COVID-19 $\cdot$ Survival $\cdot$ MELD score

\section{Introduction}

There has been a debilitating impact of the corona virus disease 2019 (COVID-19) pandemic on healthcare systems across the

Abu Bakar Hafeez Bhatti

Abubakar.hafeez@yahoo.com; abubakar.hafeez@ shifa.com.pk

1 Department of Hepato-Pancreatico-Biliary Surgery and Liver Transplantation, Shifa International Hospital Islamabad, Islamabad, Pakistan

2 Shifa Tameer-e-Millat University, Islamabad, Pakistan

3 Department of Hepatology, Shifa International Hospital Islamabad, Islamabad, Pakistan

4 Department of Surgical Critical Care, Shifa International Hospital Islamabad, Islamabad, Pakistan

5 Department of Anesthesiology, Shifa International Hospital Islamabad, Islamabad, Pakistan world. ${ }^{25}$ Solid organ transplantation appears to be one of the hardest hit specialties with a marked reduction in transplant volume. Patients undergoing liver transplantation (LT) remain a vulnerable group with immune compromise, risk of postoperative infections, and surgical complications. ${ }^{13}, 18,25$ Living donor liver transplantation (LDLT) entails additional concerns related to donor safety and well-being in the times of pandemic. Consequentially, many transplant societies have proposed that LDLT should be limited to high-risk patients. ${ }^{10} 19$ While LDLT might be an elective procedure in the west, it is the only mode of transplantation for very sick patients in many Asian countries, and relies heavily on hospital systems able to carry out complex surgeries safely. It is hypothesized that outcomes of LDLT will be compromised due to overburdened hospital systems during the COVID-19 pandemic. $^{14}$ Moreover, as potential donors and transplant candidates recover from COVID-19 infection, the safety and timing of LT in these individuals remain unknown. 
In Pakistan, the first case of COVID-19 was detected on 26th February 2020. At Shifa International Hospital Islamabad, LDLT activity was put on hold on 15 th March 2020, until specific protocols were in place to ensure safe LT. Here we share our experience with LDLT during the pandemic and its impact on transplant volumes and outcomes.

\section{Materials and Methods}

We reviewed patients who underwent LDLT between 1 st January 2019 and 31st January 2021. A total of 215 transplants were performed during this period. Details of donor and recipient selection, their evaluation, and listing process have been detailed elsewhere. ${ }^{6} 7$

\section{LDLT During the COVID-19 Pandemic}

In Pakistan, the first case of COVID-19 was reported on 26th February 2019and Shifa International Hospital remained open for COVID-19 patients. The transplant program was put on hold on 15th March 2020. After a dedicated COVID pathway was established (Fig. 1), LT activity resumed in May 2020. All potential donors and recipients underwent COVID-19 PCR testing before workup was initiated. As per Human Organ and Transplant Authority of Pakistan, it was mandatory for both donors and recipients to be COVID-19 PCR negative before transplant approval was granted. In addition, COVID-19 PCR was repeated 2 days prior to LT and a high-resolution chest CT was performed 1 day prior for all donors and recipients. If negative for COVID-19 PCR, both donors and patients were isolated in the hospital. Patients and donors with a positive COVID-19 PCR or typical/intermediate findings of COVID-19 on CT chest were delayed until PCR was negative and CT chest was negative. All asymptomatic patients and potential donors, with a positive COVID-19 PCR, were encouraged to isolate themselves, and repeat testing was performed in 3 weeks. No major changes were made to our standard immunosuppression (calcineurin inhibitors and low-dose steroids), antibiotics, and anti-coagulation protocols during the COVID-19 pandemic. We did not perform routine COVID-19 PCR in the posttransplant period unless it was clinically indicated. The posttransplant outpatient consultation was predominantly via our Telehealth system unless it was clinically necessary to see the patients in

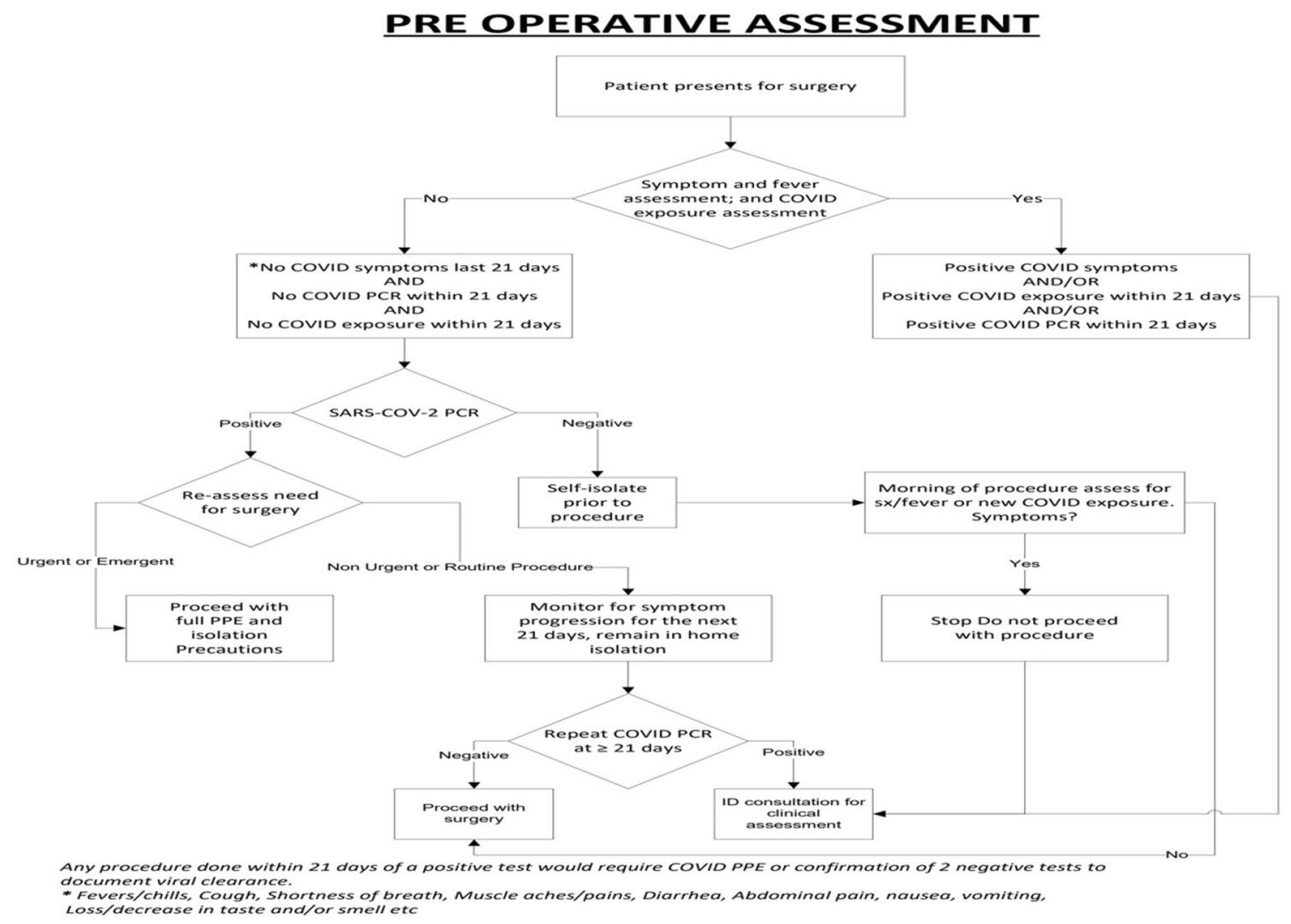

Fig. 1 Preoperative assessment protocol for COVID-19 at Shifa International Hospital Islamabad 
person. Since most of our patients reside in far off cities, they were encouraged to arrange accommodation in the hospital vicinity for at least 3 weeks after discharge.

\section{Study Design}

For this study, we divided our patients to pre-COVID (January 2019-March 2020) and COVID (April 2020Janaury 2021) cohorts. We reviewed demographics, etiology, MELD scores, presence of hepatocellular carcinoma (HCC), various graft, and operative variables. The primary outcome of interest was 30-day mortality and major morbidity in transplanted patients and donors. Morbidity was graded based on Clavien-Dindo grading and all major (grade 3 and above) complications were recorded.

\section{Statistical Analysis}

Categorical variables were represented as frequencies and percentages. Interval data was represented as means with standard deviation (SD) or medians with inter-quartile range (IQR) depending on distribution of data. Chisquare and Fischer test were used for categorical variables while $t$ test or Mann Whitney $U$ test was used for interval variables. Kaplan-Meier curves were used to determine estimated 1-year overall survival (OS) for the entire cohort (January 2019-August 2020) and Log rank test was used to determine significance. A P value $<0.05$ was considered statistically significant. All analyses were performed on Statistical Package for Social Sciences (SPSS) version 20. This study was approved by the hospital ethics committee (IRB\# 062-21).

\section{Results}

Table 1 shows the patient characteristics in the two cohorts. Fewer MELD $>20$ patients underwent LDLT in the COVID group $(41.3 \%$ versus $24.5 \%, \mathrm{P}=0.03)$. Between March and December 2020, the three monthly percentage of patients undergoing LDLT with a MELD > 20 remained under 30\% (Fig. 2). The estimated 1-year OS with a MELD score cutoff of 20 was $90 \%$ and $84 \%(P=0.2)$ as shown in Fig. 3.

There was no significant difference in 30-day patient survival $(93.2 \%$ versus $90.6 \%, \mathrm{P}=0.3$ ) (Table 2 ). More patients underwent multiple hepatic venous anastomoses $(78.3 \%$ versus $96.3 \%, \mathrm{P}=0.002)$ in the COVID cohort. A significant increase in warm ischemia time (46 min versus $56 \mathrm{~min}, \mathrm{P}=$ $0.005)$ and duration of surgery $(9 \mathrm{~h}$ versus $11 \mathrm{~h}, \mathrm{P}<0.001)$ was seen in the COVID cohort. There were nine (4.1\%) grade 3 complications in donors (re exploration for bleeding $=1$, ERCP for bile leak $=1$, image-guided percutaneous drain placement for abdominal collections $=7$ ). There was no donor mortality.

Figure 4 shows the transplant workload during the COVID-19 pandemic. There were 66 donors and recipients undergoing workup between March 2020 and January 2021 . There were 13/66 (19.6\%) deaths during transplant workup on waiting list (post TACE liver failure $=2$, liver-related decompensations $=9$, COVID-19 pneumonia $=2$ ). There were 9 patients and 3 donors who had a positive COVID-19 PCR
Table 1 Patient characteristics in the pre-COVID and COVID cohorts

\begin{tabular}{llll}
\hline & Pre-COVID cohort $(\mathrm{n}=162)$ & COVID cohort $(\mathrm{n}=53)$ & P value \\
\hline Recipient age, mean \pm SD & $45.5 \pm 14.1$ & $47.6 \pm 11.6$ & 0.3 \\
Gender, males, $\mathrm{n}(\%)$ & $130(80.2)$ & $45(84.9)$ & 0.5 \\
MELD score, median (IQR) & $18(12.7-24)$ & $16(10.5-19.5)$ & 0.2 \\
MELD groups, $\mathrm{n}(\%)$ & & $11(20.7)$ & 0.1 \\
$\leq 10$ & $19(11.8)$ & $29(54.7)$ & \\
$11-19$ & $76(46.9)$ & $10(18.9)$ & 0.03 \\
$20-29$ & $53(32.7)$ & $3(5.7)$ & 0.7 \\
$\geq 30$ & $14(8.6)$ & $13(24.5)$ & \\
MELD $>20$ & $67(41.3)$ & & \\
Etiology, $\mathrm{n}(\%)$ & & $28(52.8)$ & \\
HCV infection & $66(40.7)$ & $9(17)$ & \\
HBV infection & $23(14.2)$ & $9(17)$ & 0.7 \\
HBV and HDV infection & $25(15.4)$ & $6(11.3)$ & 0.8 \\
Cryptogenic & $34(21)$ & $1(1.9)$ & $20(37.7)$ \\
Others & $14(8.6)$ & $8(15.1)$ & \\
Hepatocellular carcinoma, $\mathrm{n}(\%)$ & $57(35.1)$ & $26(16)$ &
\end{tabular}


Fig. 2 Percentage of high MELD $(>20)$ patients who underwent LDLT at our center before and during the COVID-19 pandemic

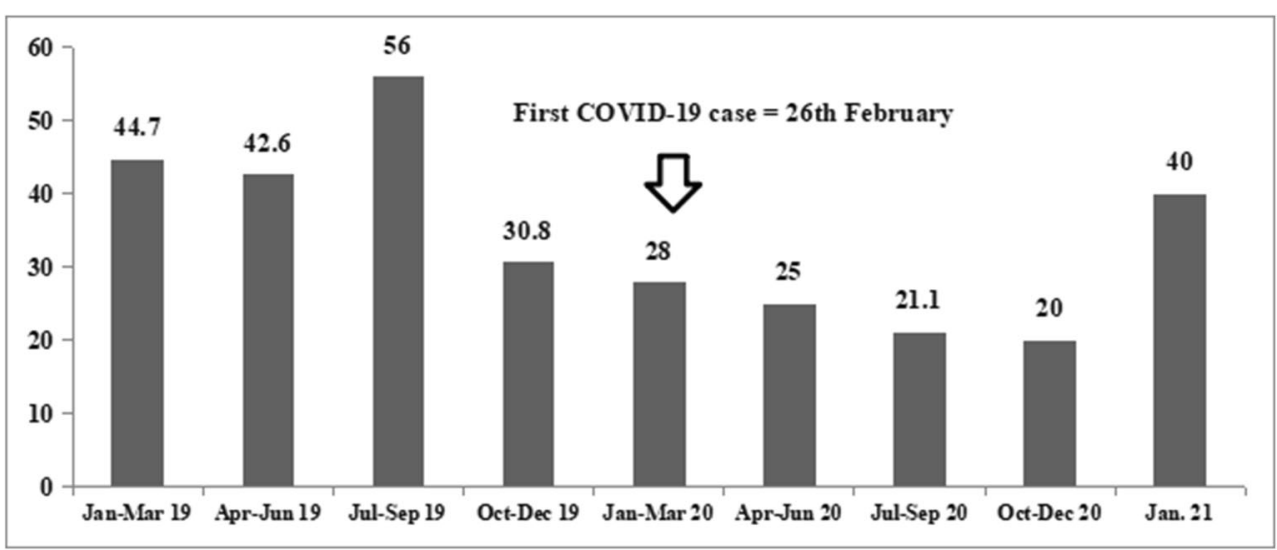

during pretransplant workup. There were 2/9 (22.2\%) mortalities due to COVID-19 pneumonia in patients on the waiting list. The remaining seven patients underwent LDLT, with one posttransplant mortality due to biliary sepsis. All three donors were asymptomatic, proceeded to donation, and had no adverse events in the postoperative period. Two patients out of 53 who underwent LDLT tested positive for COVID-19 PCR in the second week posttransplant. Both patients were negative for COVID-19 in the pretransplant workup. COVID-19 PCR and CT chest were repeated in the postoperative period due to respiratory symptoms. One out of these two patients expired on postoperative day 27 while the other patient continues to be in critical care on high-flow oxygen.

\section{Discussion}

The current study reports outcomes of LDLT during the COVID-19 pandemic from an exclusively LDLT center in Pakistan. There was a marked reduction in overall transplant volume, specifically for sick patients. Although overall short-

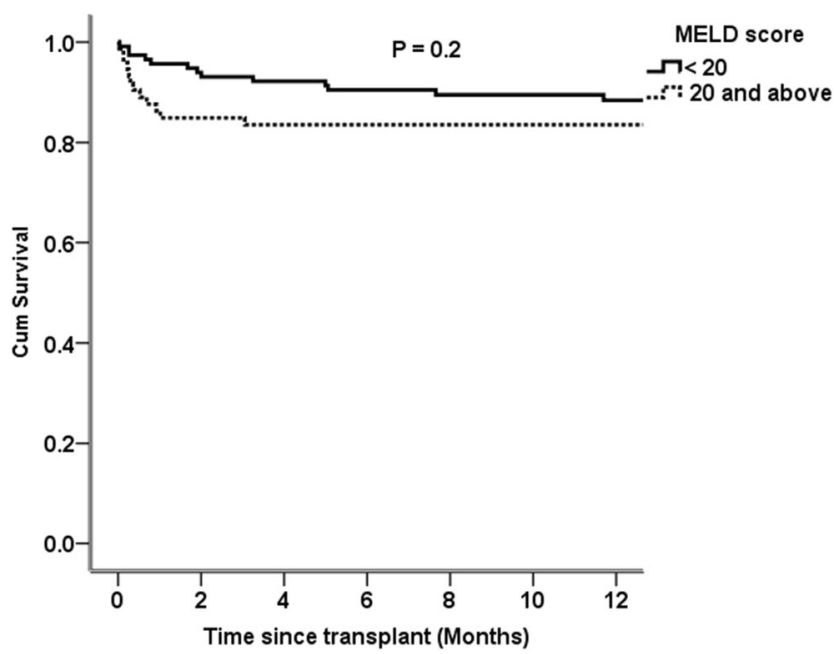

Fig. 3 Estimated 1-year overall survival for a MELD score cutoff of 20 (patients transplanted between January 2019 and August 2020) term outcomes were similar, COVID-19 infection in the early posttransplant period appeared to be associated with negative outcomes.

Various transplant societies have proposed that LDLT should be limited to sick patients during the pandemic. ${ }^{3}, 8,10,16,17,19,22$ We used a MELD cutoff of 20 to define sicker patients in the current study. It is well recognized that 3-month mortality increases from 6 to $20 \%$ with a MELD cutoff of 20. We believe that MELD $>20$ patients need prioritization for LT during the pandemic. ${ }^{24}$ Despite our attempts to transplant sicker patients first, there was a significant reduction in MELD > 20 patients who underwent LDLT. We believe that this finding might be unique to our center and reflects upon peculiar geographic and infrastructure-related factors in Pakistan. There are very few active LDLT programs in Pakistan, and most patients travel to our center from other major cities. ${ }^{12}$ During the pandemic, it was increasingly difficult for sicker patients to fly across cities and secure admission in critical care units for urgent care. Table 3 shows the factors that might have led to a reduction in LDLT for sick patients in the COVID cohort.

As the COVID-19 pandemic prolongs, there are concerns regarding the risks of re infection in potential donors and transplant candidates. "The safe window in which such patients and potential donors are most suited to undergo LT remains unknown. It has been recommended that living donors should self-quarantine for 2-3 weeks before donation. Moreover, if they have a positive COVID-19 PCR, they should wait for 4 weeks after symptom resolution and proceeding to donation. ${ }^{9}$ All three donors and seven recipients in our study, who underwent LDLT, had asymptomatic COVID-19 infection and were positive on screening PCR which was part of the transplant workup. As per our hospital protocol, we waited 3 weeks before repeating COVID-19 PCR in asymptomatic individuals with an initial positive PCR. If the PCR was negative, CT chest was also performed before proceeding with LT. None of our patients and donors had repeat COVID-19 infection in the follow-up period. 
Table 2 Thirty-day recipient outcomes in the pre-COVID and COVID cohorts

\begin{tabular}{|c|c|c|c|}
\hline & $\begin{array}{l}\text { Pre-COVID cohort } \\
(\mathrm{n}=162)\end{array}$ & COVID cohort $(n=53)$ & $\mathrm{P}$ value \\
\hline 30-day patient survival, n (\%) & $151(93.2)$ & $48(90.6)$ & 0.3 \\
\hline 30-day patient survival-MELD score, $\mathrm{n}(\%)$ & & & 0.5 \\
\hline MELD $<20(n=135)$ & $91 / 95(95.7)$ & $38 / 40(95)$ & \\
\hline MELD $\geq 20(n=80)$ & $60 / 67(89.6)$ & 10/13 (76.9) & \\
\hline Cause of death, $n$ & & & 0.2 \\
\hline Graft dysfunction & 2 & 2 & \\
\hline Vascular event & 3 & - & \\
\hline Biliary complication & 1 & 1 & \\
\hline Myocardial infarction & 1 & - & \\
\hline Sepsis & 2 & - & \\
\hline Gastric bleed & 1 & - & \\
\hline Cerebrovascular event & 1 & 1 & \\
\hline COVID-19 pneumonia & 0 & 1 & \\
\hline Hepatic artery thrombosis, $\mathrm{n}(\%)$ & 5 & 0 & 0.3 \\
\hline Portal vein thrombosis, $\mathrm{n}(\%)$ & 2 & 0 & 1 \\
\hline Biliary complications, n (\%) & $10(6.2)$ & $3(5.7)$ & 1 \\
\hline Graft dysfunction, n (\%) & $22(13.5)$ & $2(3.8)$ & 0.07 \\
\hline Re exploration, $\mathrm{n}(\%)$ & $6(3.7)$ & $1(1.9)$ & 1 \\
\hline ICU stay, days, median (IQR) & $5(4-6)$ & $5(4-6)$ & 0.2 \\
\hline Hospital stay, days, median (IQR) & $16(13.7-18)$ & $15.5(13.7-18)$ & 0.6 \\
\hline$>1$ biliary anastomoses, $\mathrm{n}(\%)$ & $39(24)$ & $19(35.8)$ & 0.1 \\
\hline$>1$ hepatic vein anastomoses, $\mathrm{n}(\%)$ & $127(78.3)$ & $51(96.3)$ & 0.002 \\
\hline Actual GRWR < 0.8, n (\%) & $32(19.7)$ & $6(11.3)$ & 0.1 \\
\hline Cold ischemia time (min), median (IQR) & $50(30-73.5)$ & $52(42.5-67.5)$ & 0.6 \\
\hline Warm ischemia time (min), median (IQR) & $46(35-56)$ & $56(46.5-69.5)$ & 0.005 \\
\hline Blood loss (ml), median (IQR) & $1400(837-2025)$ & $1500(1000-2500)$ & 0.6 \\
\hline Surgery duration, hours, median (IQR) & $9(7-12)$ & $11(10-13)$ & $<0.001$ \\
\hline
\end{tabular}

Except one recipient who had biliary sepsis and died, all donors and recipients are doing well at the last follow-up.
Moreover, none of these donors or recipients had pulmonary complications in the follow-up period.
Fig. 4 Mortality in listed patients during the COVID-19 pandemic (March 2020-January 2021)

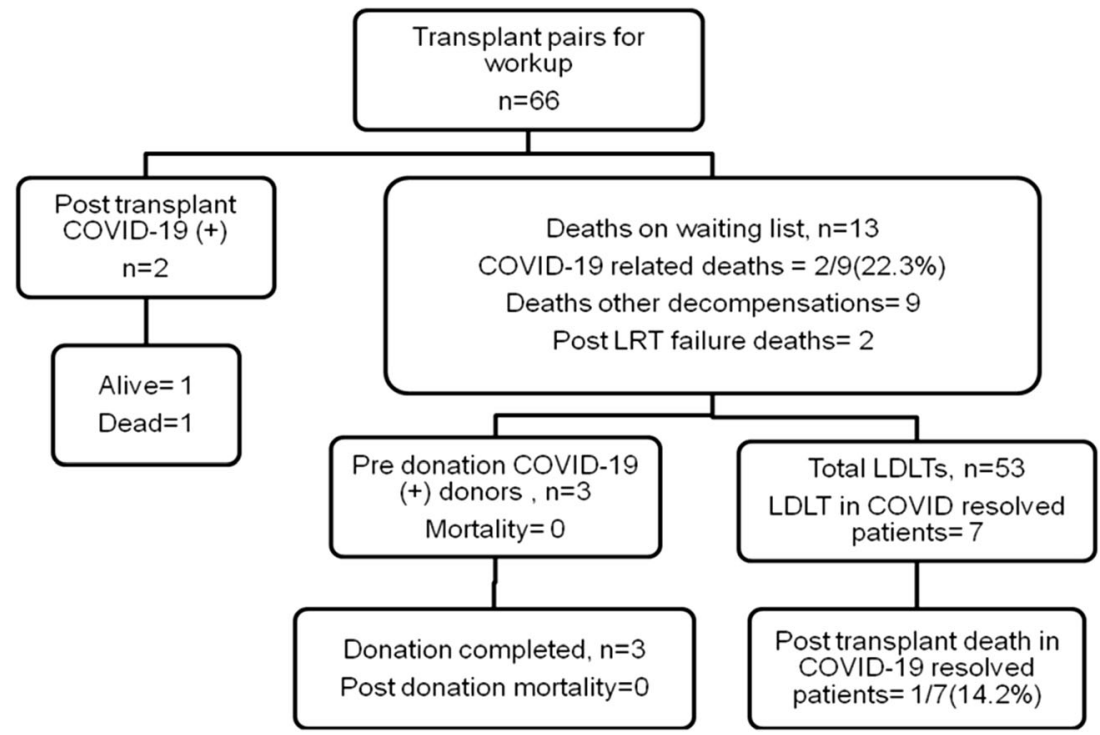


Table 3 Potential factors leading to delays in transplanting sicker patients during the COVID-19 pandemic

Transportation from other cities due to restrictions on air travel and ground transport

Overburdened intensive care/critical care units in transplant and non transplant centers

Non availability of trained staff during the pandemic in intensive care units, operating rooms, and floors

Telehealth systems inadequate to cater for sick patients needing on site evaluation

Delays in access to supportive treatments like endoscopy and interventional radiology with a prerequisite COVID-19 PCR testing

Donor concerns regarding possible COVID-19 infection in the post donation period

The impact of COVID-19 infection in liver transplant recipients remains less well understood and published reports predominantly deal with patients who developed COVID-19 infection years after LT. Old age, comorbidities, and metabolic abnormalities might increase the risk of adverse events with COVID-19 infection. ${ }^{2}, 15,23$ Very few cases of early infection, especially in the first month after liver transplant, have been reported. ${ }^{1}, 21$ There were two patients with documented COVID-19 infection within 2 weeks of LT in the current study. Both these patients were negative for COVID-19 in the pretransplant workup. One patient died due to COVID19 pneumonia and the other remains in the critical care unit with high oxygen demand. In one patient, the source was traced back to the patient's close family while in the other, no obvious source could be determined. Both patients had MELD scores between 20 and 25 and no prior pulmonary problems.

Currently we are in the process of contacting all our transplanted patients, encouraging them to practice strict precautions, get vaccinated, and check COVID-19 antibody levels. In Pakistan, at this time, access to vaccination remains limited to individuals aged $>60$ years and healthcare professionals. With recent evidence suggesting significant reduction in mortality after elective surgery in vaccinated patients, we now encourage all our donors to get vaccinated if there is a 34-week anticipated delay in liver transplantation. We think that in time, we should see improvement in donation rates based upon the encouraging results from the international COVIDsurg collaboration report.

There are certain limitations of the current study. Since our hospital was open to COVID-19-positive patients, our protocol and findings might not be applicable to LDLT centers that remain COVID-free centers. Moreover, the primary objective of the current study was to assess the impact of COVID-19 on immediate posttransplant outcomes. The actual 1-year survival and long-term results in patients transplanted during the pandemic merit further exploration but need more time. For survival analysis, we included patients with at least 6-month follow-up and showed an acceptable estimated 1-year survival. The number of donors and recipients who underwent LDLT after recovery from COVID-19 infection is rather low to draw meaningful conclusions, but the results appear promising.

\section{Conclusions}

The current study demonstrates comparable short-term LDLT outcomes in the pre-COVID and COVID cohorts. It underscores the challenges in transplanting sicker patients during the pandemic due to limitations in infrastructure, increasingly overwhelmed healthcare facilities, and impending threats of COVID-19 infection in the early post LT period. With accumulating data, it is important to promote data sharing between centers to assess safety of LDLT during the pandemic and assess its short-term and long-term impact on transplant outcomes.

Author Contributions AHB contributed to the concept, design, data analysis, writing, and critical review of the manuscript.

$\mathrm{MN}$ and NYK contributed to the concept, design, and data collection.

FM and HHZ contributed to the design, manuscript writing, and critical review of the manuscript.

AI, WI, and NAK contributed to the concept and critical review of the manuscript.

\section{Declarations}

Conflict of Interest The authors declare no competing interests.

\section{References}

1. Bhatti ABH, Riyaz S, Akhtar A. In-hospital Mortality after Liver Transplantation due to COVID-19. J Coll Physicians Surg Pak 2020 Oct;30(10):141-142.

2. Bhoori S, Rossi RE, Citterio D, Mazzaferro V. COVID-19 in longterm liver transplant patients: preliminary experience from an Italian transplant centre in Lombardy. Lancet Gastroenterol Hepatol 2020;5(6):532-533.

3. Boyarsky BJ, Po-Yu Chiang T, Werbel WA, et al. Early impact of COVID-19 on transplant center practices and policies in the United States. Am J Transplant 2020;20(7):1809-1818.

4. Clavien PA, Barkun J, de Oliveira ML, Vauthey JN, Dindo D, Schulick RD, et al. The Clavien-Dindo classification of surgical complications: five-year experience. Ann Surg 2009;250(2):18796.

5. COVIDSurg Collaborative, GlobalSurg Collaborative. SARSCoV-2 vaccination modelling for safe surgery to save lives: data from an international prospective cohort study. Br J Surg. 2021 24: znab101. doi: https://doi.org/10.1093/bjs/znab101. Epub ahead of print. PMID: 33761533; PMCID: PMC7995808.

6. Dar FS, Bhatti AB, Dogar AW, Zia H, Amin S, Rana A, et al. The travails of setting up a living donor liver transplant program: 
Experience from Pakistan and lessons learned. Liver Transpl 2015;21(7):982-90.

7. Dar FS, Bhatti ABH, Qureshi AI, Khan NY, Eswani Z, Zia HH, Khan EU, Khan NA, Rana A, Shah NH, Salih M, Nazer R. Living Donor Liver Transplantation in South Asia: Single Center Experience on Intermediate-Term Outcomes. World J Surg 2018;42(4):1111-1119.

8. de Vries APJ, Alwayn IPJ, Hoek RAS, van den Berg AP, Ultee FCW, Vogelaar SM. Immediate impact of COVID-19 on transplant activity in The Netherlands. Transpl Immunol 2020;61:101304.

9. Di Maira T, Berenguer M. COVID-19 and liver transplantation. Nat Rev Gastroenterol Hepatol 2020;17(9):526-528.

10. Fix OK, Hameed B, Fontana RJ, Kwok RM, McGuire BM, Mulligan DC. Clinical best practice advice for hepatology and liver transplant providers during the COVID-19 pandemic: AASLD expert panel consensus statement. Hepatology. 2020;72:287-304

11. Gousseff M, Penot P, Gallay L, Batisse D, Benech N, Bouiller K, et al; in behalf of the COCOREC study group. Clinical recurrences of COVID-19 symptoms after recovery: Viral relapse, reinfection or inflammatory rebound? J Inf Secur 2020;81(5):816-846.

12. Hafeez Bhatti AB, Saud Dar F. Living Donor Liver Transplantation in Pakistan. Transplantation. 2017;101(7):1507-1508.

13. Huang JF, Zheng KI, George J, Gao HN, Wei RN, Yan HD, et al. Fatal outcome in a liver transplant recipient with COVID-19. Am J Transplant 2020;20(7):1907-10.

14. Jha SK, Jamir I, Sisodia K, Kumar N, Sood G, Shanker N, et al. Restarting LDLT During COVID-19: Early Results After Restructuring. Transplant Proc. 2020 16:S0041-1345(20)32902-X.

15. Lee BT, Perumalswami PV, Im GY, Florman S, Schiano TD; COBE Study Group. COVID-19 in Liver Transplant Recipients: An Initial Experience From the US Epicenter. Gastroenterology. 2020;159(3):1176-1178.e2.

16. Maggi U, De Carlis L, Yiu D, Colledan M, Regalia E, Rossi G, et al. The impact of the COVID-19 outbreak on liver transplantation programs in northern Italy. Am J Transplant 2020;20(7):1840-1848

17. Pan L, Zeng J, Yang H. Challenges and countermeasures for organ donation during the SARS-CoV-2 epidemic: the experience of
Sichuan Provincial People's Hospital. Intensive Care Med 2020;46(5):844-845.

18. Pereira MR, Mohan S, Cohen DJ, Husain SA, Dube GK, Ratner LE, et al. COVID-19 in solid organ transplant recipients: Initial report from the US epicenter. Am J Transplant 2020;20(7):1800-8.

19. Saigal S, Gupta S, Sudhindran S, Goyal N, Rastogi A, Jacob M, et al. Liver transplantation and COVID-19 (Coronavirus) infection: guidelines of the liver transplant Society of India (LTSI). Hepatol Int 2020;14(4):429-431. doi: https://doi.org/10.1007/s12072-02010041-1.

20. Soin AS, Choudhary NS, Yadav SK, Saigal S, Saraf N, Rastogi A, et al. Restructuring Living Donor Liver Transplantation at a High Volume Center During the COVID-19 Pandemic. J Clin Exp Hepatol 2020. doi: https://doi.org/10.1016/j.jceh.2020.09.009.

21. Umberto M., Luciano D.C., Daniel Y. The impact of the COVID19 outbreak on liver transplantation programs in Northern Italy. Am J Transplant 2020;20:1840-1848.

22. Wang Y, Yang H, Liu H, Buhler LH, Deng S. Strategies to halt 2019 novel coronavirus (SARS-CoV-2) spread for organ transplantation programs at the Sichuan Academy of Medical Science and Sichuan Provincial People's Hospital, China, Am J Transplant 2020;20(7):1837-1839.

23. Webb GJ, Moon AM, Barnes E, Barritt AS, Marjot T. Determining risk factors for mortality in liver transplant patients with COVID19. Lancet Gastroenterol Hepatol 2020;5(7):643-644.

24. Wiesner R, Edwards E, Freeman R, Harper A, Kim R, Kamath P, et al; United Network for Organ Sharing Liver Disease Severity Score Committee. Model for end-stage liver disease (MELD) and allocation of donor livers. Gastroenterology. 2003;124(1):91-6.

25. Yi SG, Rogers AW, Saharia A, Aoun M, Faour R, Abdelrahim M, et al. Early Experience With COVID-19 and Solid Organ Transplantation at a US High-volume Transplant Center. Transplantation. 2020;104(11):2208-2214.

Publisher's Note Springer Nature remains neutral with regard to jurisdictional claims in published maps and institutional affiliations. 